\title{
Effects of dynamic oxygen concentrations on the development of mouse pre- and peri-implantation embryos using a double-channel gas supply incubator system
}

\author{
Seung-Chan Lee', Ho-Chul Seo', Jaewang Lee ${ }^{2,3}$, Jin Hyun Jun ${ }^{2,3,4}$, Kyoo Wan Choi' \\ ${ }^{1}$ CNC Biotech Inc., Suwon; ${ }^{2}$ Department of Biomedical Laboratory Science, Eulji University, Seongnam; Departments of ${ }^{3}$ Biomedical Laboratory Science \\ and ${ }^{4}$ Senior Healthcare, BK21 PLUS Program, Graduate School, Eulji University, Seongnam, Korea
}

Objective: We aimed to evaluate the effects of different oxygen conditions ( $20 \%$ [high $\mathrm{O}_{2}$ ], $5 \%$ [low $\mathrm{O}_{2}$ ] and $5 \%$ decreased to $2 \%$ [dynamic $\mathrm{O}_{2}$ ]) on mouse pre- and peri-implantation development using a novel double-channel gas supply (DCGS) incubator (CNC Biotech Inc.) to alter the oxygen concentration during in vitro culture.

Methods: The high- $\mathrm{O}_{2}$ and low- $\mathrm{O}_{2}$ groups were cultured from the one-cell to the blastocyst stage under $20 \%$ and $5 \%$ oxygen concentrations, respectively. In the dynamic- $\mathrm{O}_{2}$ group, mouse embryos were cultured from the one-cell to the morula stage under $5 \% \mathrm{O}_{2}$ for 3 days, followed by culture under $2 \% \mathrm{O}_{2}$ to the blastocyst stage. To evaluate peri-implantation development, the blastocysts from the three groups were individually transferred to a fibronectin-coated dish and cultured to the outgrowth stage in droplets.

Results: The blastocyst formation rate was significantly higher in the low- $\mathrm{O}_{2}$ and dynamic- $\mathrm{O}_{2}$ groups than in the high- $\mathrm{O}_{2}$ group. The total cell number was significantly higher in the dynamic- $\mathrm{O}_{2}$ group than in the low- $\mathrm{O}_{2}$ and high $-\mathrm{O}_{2}$ groups. Additionally, the apoptotic index was significantly lower in the low- $\mathrm{O}_{2}$ and dynamic- $\mathrm{O}_{2}$ groups than in the high- $\mathrm{O}_{2}$ group. The trophoblast outgrowth rate and spread area were significantly higher in the low- $\mathrm{O}_{2}$ and dynamic- $\mathrm{O}_{2}$ groups than in the high- $\mathrm{O}_{2}$ group.

Conclusion: Our results showed that a dynamic oxygen concentration (decreasing from $5 \%$ to $2 \%$ ) had beneficial effects on mouse pre- and peri-implantation development. Optimized, dynamic changing of oxygen concentrations using the novel DCGS incubator could improve the developmental competence of in vitro cultured embryos in a human in vitro fertilization and embryo transfer program.

Keywords: Apoptosis; Blastocyst; In vitro fertilization; Oxygen; Outgrowth

Received: August 12, 2019 Revised: September 10, 2019.

Accepted: September 24, 2019

Corresponding author: Jin Hyun Jun

Department of Biomedical Laboratory Science, Eulji University, 553 Sanseong-

daero, Sujeong-gu, Seongnam 13135, Korea

Tel:+82-31-740-7210 Fax:+82-31-740-7354 E-mail:junjh55@hanmail.net

*This work was supported by the Business for Cooperative R\&D between Industry, Academy, and Research Institute, funded by the Korea Small and Medium Business Administration in 2018 (Grants No. 17-05).

This is an Open Access article distributed under the terms of the Creative Commons Attribution Non-Commercial License (http://creativecommons.org/licenses/by-nc/4.0/) which permits unrestricted non-commercial use, distribution, and reproduction in any medium, provided the original work is properly cited.

\section{Introduction}

Oxygen is an essential physiological component for regulating embryonic development in the environments of the oviduct and the uterus. Oxygen concentration impacts the rate of embryonic development and the quality of in vitro culture. The majority of modern in vitro fertilization (IVF) labs have accepted a standard of either $5 \%$ or $20 \%$ oxygen. In the early years of IVF, the embryo was cultured at atmospheric oxygen concentrations (20\%); this was proven effective by the birth of millions of children conceived using this method $[1,2]$. 
However, many studies have reported that the detrimental effects of oxidative stress during embryonic development at atmospheric oxygen concentrations (20\%) were related to an increase in reactive oxygen species (ROS) [3-5]. ROS have been implicated in the damaged development of mammalian embryos in in vitro culture [6]. Exposure to these species causes DNA fragmentation and irreversible doublestrand breaks, which may be especially frequent in the early embryonic stage of development due to active DNA replication during this time. Cellular damage caused by ROS induces uneven embryo cleavage, delayed cleavage, and developmental arrest of the embryo [7]. In addition, hydrogen peroxide, an example of an ROS, is the main mediator of apoptosis and cytoplasmic fragmentation in blastocysts [8].

The physiological oxygen concentration in the mammalian oviduct is around 5\% [9-11]. As early as the early 1970s, Steptoe et al. [12] reported that human embryonic development occurred successfully and even improved when using an oxygen concentration of $5 \%$. Other studies have also demonstrated beneficial effects of a $5 \%$ oxygen concentration on mammalian embryonic development [13-16]. Furthermore, in mice, an increased blastocyst cell number and improved fetal development have been reported when embryos were cultured in an oxygen concentration of 5\% [17-21]. Although most reports have shown that using hypoxic conditions improves embryonic development, the optimal oxygen concentration for developmental stage-specific preimplantation embryos during in vitro culture has not been fully established.

Recently, new suggestions have emerged regarding whether a further reduction of oxygen concentration on day 3 after fertilization represents a physiological condition that is more similar to in vivo conditions during development [11]. These new approaches were based on the premise that the oxygen concentration is actually lower in the uterus than in the oviduct. As the embryo reaches the uterus, it experiences a decrease in oxygen concentration, measured at around $2 \%$, whereas the oxygen concentration in the oviduct is around $5 \%$ $7 \%$, at least in nonhuman species [22,23]. Thompson et al. [24] reported that bovine embryonic development was improved by reducing the oxygen concentration from $7 \%$ to $2 \%$ at the post-compaction stage. Such an oxygen gradient is similar to that in the female reproductive tract, which has a relatively high oxygen concentration in the oviducts (5\%-8\%) and a very low oxygen concentration in the uterus (approximately 2\%). Researchers have attempted to mimic the in vivo environment using an in vitro culture system to study preimplantation embryonic development; however, more research is needed to confirm the hypothesis that we previously described.

In the present study, we hypothesized that sequential exposure to a $5 \%$ oxygen concentration from day 1 to day 3 (the pre-compaction stage), and then to a $2 \%$ oxygen concentration from day 3 to day 5 (the post-compaction stage), may improve developmental compe- tence compared with continuous exposure to either a $5 \%$ or a $20 \%$ oxygen concentration. This study was performed to evaluate the effects of dynamic conditions using a novel double-channel gas supply (DCGS) incubator (CNC Biotech Inc., Suwon, Korea) on mouse preand peri-implantation development.

\section{Methods}

\section{Animals and hormonal stimulation}

This study was approved by the Eulji University Institutional Animal Care and Use Committee (No. EUIACUC 17-14). The protocol for superovulated mice was described by Park et al. [25]. Briefly, 6- to 9-week-old female BDF mice were superovulated with intraperitoneal injections of $5 \mathrm{IU}$ of pregnant mare's serum gonadotropin (Prospec, Rehovot, Israel), and 48 hours later, the mice were injected with $5 \mathrm{IU}$ of human chorionic gonadotropin (Prospec). The superovulated mice were then individually mated with a fertile male BDF mouse.

\section{Embryo collection and in vitro culture condition}

Once 19 hours had passed after mating, female mice with a confirmed vaginal plug were sacrificed by cervical dislocation, and cumulus-enclosed one-cell embryos (zygotes) were retrieved from the oviductal ampullae. Then, the zygotes were denuded by incubation for 1 minute with $0.1 \%$ hyaluronidase (Sigma-Aldrich, St. Louis, MO, USA) in phosphate-buffered saline. The zygotes were pooled and washed three times in Continuous Single Culture-NX (CSCM-NX; FUJIFILM Irvine Scientific, Santa Ana, CA, USA) with 10\% human serum albumin (HSA; Irvine). The five healthy zygotes were cultured in 10 $\mu \mathrm{L}$ of CSCM medium with $10 \%$ HSA covered with mineral oil for 4 days under three different oxygen conditions.

We used a newly developed incubator with the DCGS system (Figure 1). This incubator could set the conversion timing of different concentrations of $\mathrm{O}_{2}$ gas from $5 \%$ to $2 \%$. It is a unique characteristic of the DCGS incubator system. In the dynamic- $\mathrm{O}_{2}$ group, zygotes were cultured from the one-cell to the morula stage under $5 \% \mathrm{O}_{2}$ for 3 days, followed by culture under $2 \% \mathrm{O}_{2}$ concentration to the blastocyst stage. Embryos in the high- $\mathrm{O}_{2}$ and low- $\mathrm{O}_{2}$ groups were cultured from the one-cell to the blastocyst stage under $20 \%$ and $5 \%$ oxygen concentrations, respectively. The cleavage rate from the zygote to the two-cell stage and the development rate to the blastocyst stage were determined 24 hours and 96 hours after zygote collection, respectively.

\section{Detection of apoptosis and calculation of the apoptotic index}

Apoptotic cells were detected using a fluorescein isothiocyanateconjugated in situ cell death detection kit (terminal deoxynucleotidyl 

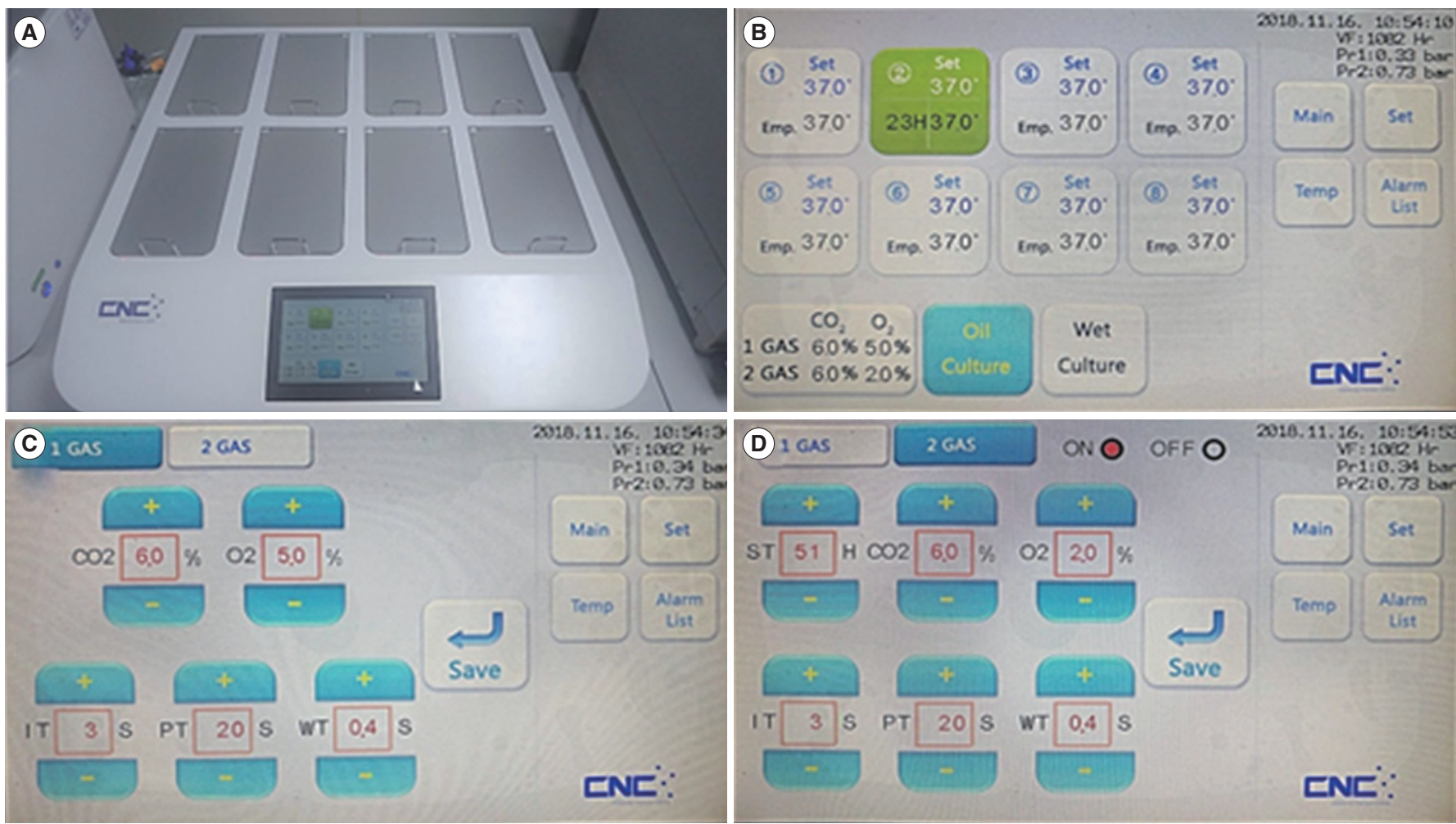

Figure 1. Newly developed incubator with a double-channel gas supply (DCGS) system. (A) Image of a DCGS incubator with eight chambers and a touch-panel screen. (B) Main screen of the DCGS incubator that displays the temperature, incubation time, and gas concentration of each chamber. (C) Screen for controlling the first gas supply of each chamber. (D) Screen for controlling the second gas supply of each chamber.

transferase dUTP nick end labeling [TUNEL]; Promega, Durham, NC, USA). The blastocysts were fixed with $4 \%$ paraformaldehyde. The apoptotic cells were stained using a TUNEL kit according to the manufacturer's instructions, and the nuclei were stained in a solution of $10 \mathrm{mg} / \mathrm{mL}$ bisbenzimide (Hoechst 33342, Sigma) for 10 minutes prior to observation with a fluorescence microscope (AX-70; Olympus, Tokyo, Japan). Additionally, the number of cells with TUNEL-positive nuclei was determined using an image capturing system (IMT i-Solution, British Columbia, Canada). The apoptotic index was calculated as the percentage of TUNEL-positive nuclei divided by the total number of nuclei in a single blastocyst.

\section{Outgrowth assay for evaluation of peri-implantation development}

The blastocysts were transferred to a fibronectin-coated dish and outgrown to examine the effects of different oxygen concentrations on peri-implantation embryonic development. The morphological changes during peri-implantation development were observed every 24 hours, and the captured images were analyzed. The area of trophoblastic outgrowth was measured after 72 hours using ImageJ software (National Institutes of Health, Bethesda, MD, USA) as described previously [26].

\section{Statistical analysis}

All experiments were performed at least in triplicate. All comparisons between groups were determined by one-way analysis of variance. Tukey honest significant difference post hoc test was used for all comparisons between groups. A $p$-value of less than 0.05 was considered to indicate statistical significance.

\section{Results}

\section{Effects of different oxygen concentrations on preimplantation embryonic development}

Mouse embryonic development rates were assessed following culture for 24 hours (constituting development to the two-cell stage) and 96 hours (constituting development to the blastocyst stage), and are presented in Table 1. No differences were found in the twocell development rate between groups. However, the blastulation rate was significantly higher in the low $-\mathrm{O}_{2}(75.7 \% \pm 2.2 \%)$ and the dynamic $-\mathrm{O}_{2}(75.6 \% \pm 4.6 \%)$ groups than in the high- $\mathrm{O}_{2}(54.0 \% \pm$ $2.3 \%)$ group $(p<0.05)$.

\section{Effects of oxygen concentration on embryo quality}

The numbers of total cells and apoptotic nuclei in the blastocysts 
Table 1. Effects of different oxygen concentrations on the development of preimplantation embryos

\begin{tabular}{|c|c|c|c|}
\hline Group & No. of zygotes & No. of cleaved embryos (\%) & No. of developed blastocysts (\%) \\
\hline $\mathrm{High} \mathrm{O}_{2}(20 \%)$ & 151 & $131(86.9 \pm 5.8)$ & $86(54.0 \pm 2.3)^{\mathrm{a})}$ \\
\hline Low $\mathrm{O}_{2}(5 \%)$ & 154 & $131(87.9 \pm 3.5)$ & $115(75.7 \pm 2.2)^{b)}$ \\
\hline Dynamic $\mathrm{O}_{2}(5 \%$ to $2 \%)$ & 159 & $148(90.1 \pm 2.0)$ & $122(75.6 \pm 4.6)^{b)}$ \\
\hline
\end{tabular}

Values in the parentheses are presented as mean \pm standard error of the mean.

a,b) Different superscripts indicate values that differ significantly at $p<0.05$.

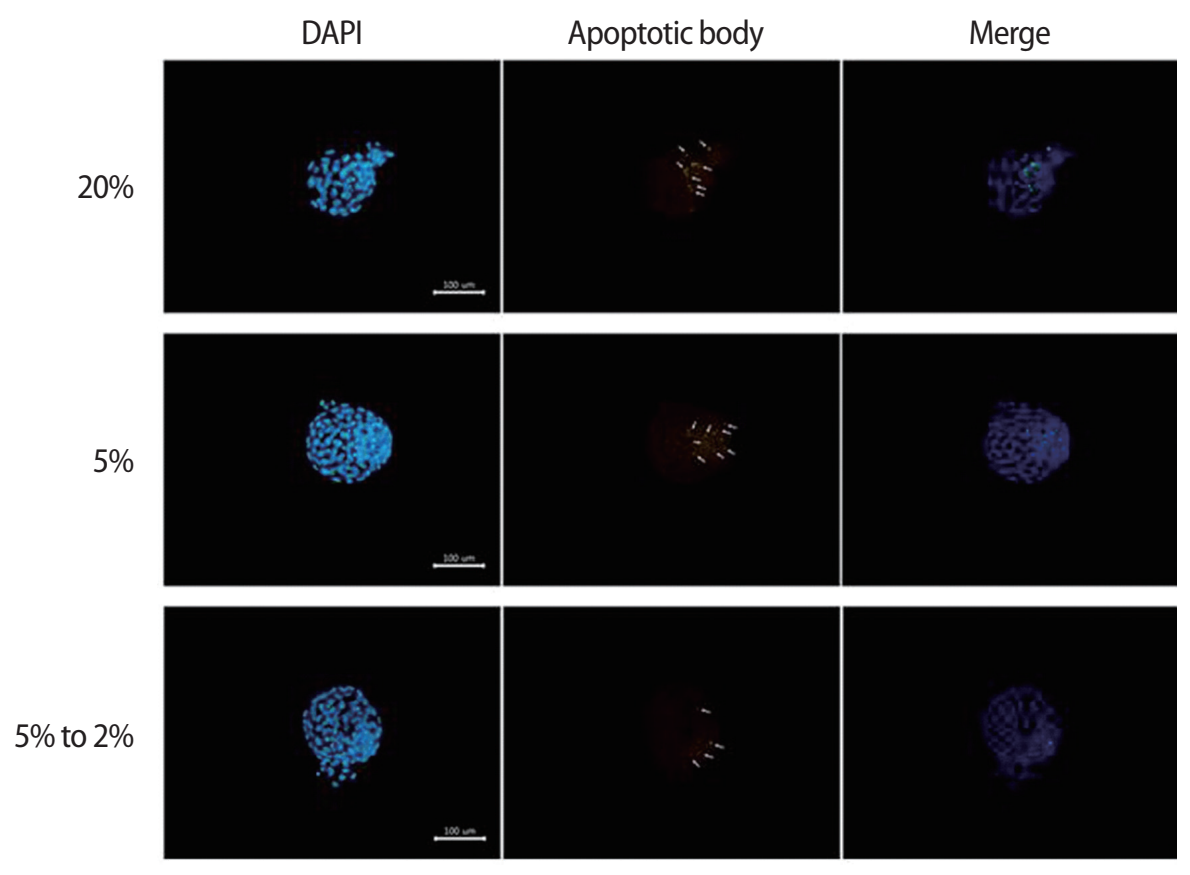

Figure 2. Assessment of apoptotic cells in blastocysts using terminal deoxynucleotidyl transferase dUTP nick end labeling assay. Fluorescence analysis for the number of total and apoptotic cells of the blastocyst. The fragmented nuclei (denoted by green fluorescence) were apoptotic (denoted by white arrows) and DNA was counterstained with the Hoechst stain (denoted by blue). Scale bar, 100 4m. DAPI, 4,6-diamidino2-phenylindole.

Table 2. Effects of different oxygen concentrations on the number of total cells and apoptotic index in blastocysts

\begin{tabular}{|c|c|c|}
\hline Group & $\begin{array}{l}\text { Total cells in } \\
\text { blastocysts }\end{array}$ & $\begin{array}{l}\text { Apoptotic index in } \\
\text { blastocysts (\%) }\end{array}$ \\
\hline $\mathrm{High} \mathrm{O}_{2}(20 \%)$ & $87.5 \pm 2.5^{\mathrm{a})}(\mathrm{n}=65)$ & $8.67 \pm 0.82^{a)}(n=50)$ \\
\hline Low $\mathrm{O}_{2}(5 \%)$ & $115.4 \pm 3.6^{b)}(n=93)$ & $\left.4.47 \pm 0.49^{b}\right)(n=83)$ \\
\hline Dynamic $\mathrm{O}_{2}$ (5\% to $\left.2 \%\right)$ & $128.9 \pm 3.3^{c)}(n=95)$ & $4.15 \pm 0.35^{b)}(n=76)$ \\
\hline
\end{tabular}

Values are presented as mean \pm standard error of the mean.

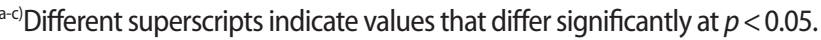

from each group were determined using Hoechst and TUNEL staining (Figure 2). The mean cell number of the blastocysts was significantly higher in the dynamic- $\mathrm{O}_{2}$ group $(128.9 \pm 3.3)$ than in the high$\mathrm{O}_{2}(87.5 \pm 2.5)$ and low $-\mathrm{O}_{2}(115.4 \pm 3.6)$ groups. In contrast, the apoptotic index was significantly lower in the dynamic- $\mathrm{O}_{2}(4.15 \% \pm 0.35 \%)$ and low $-\mathrm{O}_{2}(4.47 \% \pm 0.49 \%)$ groups than in the high $-\mathrm{O}_{2}(8.67 \% \pm$
$0.82 \%)$ group $(p<0.05)$, as shown in Table 2.

\section{Effects of different oxygen concentrations on peri- implantation embryonic development}

The developmental competence of peri-implantation embryos cultured under different oxygen concentrations was assessed using an in vitro outgrowth assay (Figure 3). After culture of blastocysts for 3 days, the outgrowth rate of the low- $\mathrm{O}_{2}(58.3 \% \pm 2.3 \%)$ and dynamic$\mathrm{O}_{2}(71.3 \% \pm 6.5 \%)$ groups was significantly higher than that of the high $-\mathrm{O}_{2}(38.4 \% \pm 0.5 \%)$ group. Correspondingly, the trophoblastic spread area in the dynamic- $\mathrm{O}_{2}\left(1.11 \pm 0.07 \mathrm{~mm}^{2}\right)$ and low $-\mathrm{O}_{2}(1.05 \pm$ $1.11 \mathrm{~mm}^{2}$ ) groups was significantly wider than that in the high- $\mathrm{O}_{2}$ $\left(0.70 \pm 0.05 \mathrm{~mm}^{2}\right)$ group $(p<0.05)$, as shown in Table 3 . 
$20 \%$

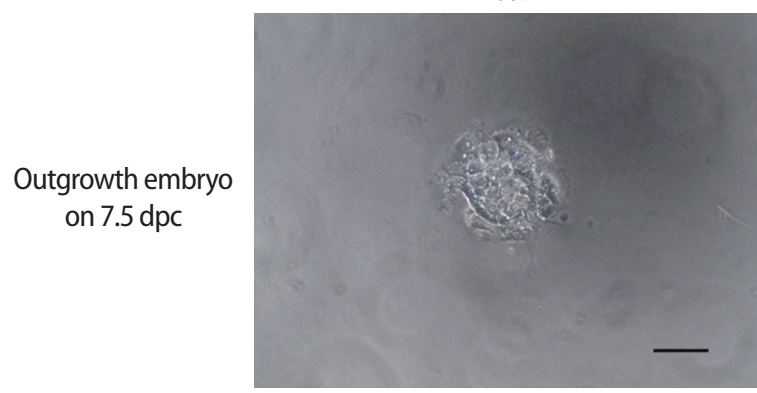

$5 \%$

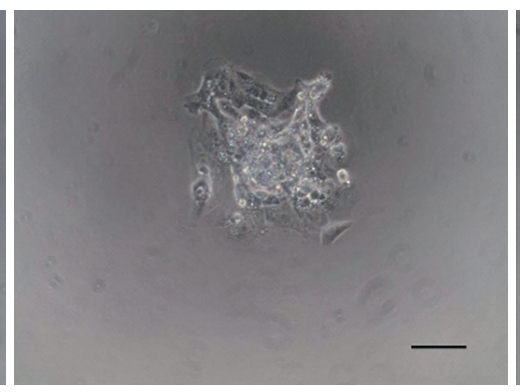

$5 \%$ to $2 \%$

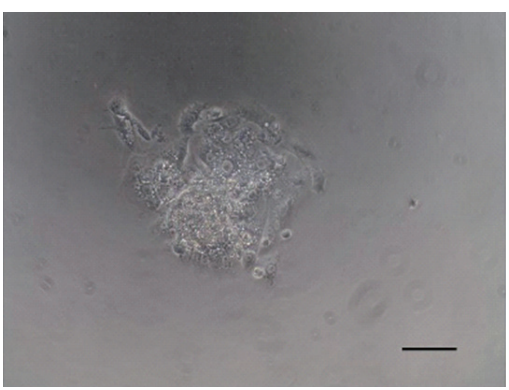

Figure 3. Effects of different oxygen concentrations on outgrowth embryos after 3-day culture of blastocysts from different groups. Morphologic assessment of outgrowth embryos under high (20\%), low (5\%), and dynamic (5\% decreasing to $2 \%$ ) oxygen concentrations. Scale bar, $100 \mu \mathrm{m}$.

Table 3. Effects of different oxygen concentrations on development of peri-implantation embryos

\begin{tabular}{lcc}
\hline Group & $\begin{array}{c}\text { No. of outgrowth embryos } \\
\text { from zygotes (\%) }\end{array}$ & $\begin{array}{c}\text { Mean spread area of } \\
\text { trophoblast cells }\left(\mathrm{mm}^{2}\right)\end{array}$ \\
\hline High $\mathrm{O}_{2}(20 \%)$ & $34 / 80(38.4 \pm 0.5)^{\mathrm{a})}$ & $0.70 \pm 0.05^{\mathrm{a})}$ \\
${\text { Low } \mathrm{O}_{2}(5 \%)}^{\text {Dynamic }} \mathrm{O}_{2}(5 \%$ to $2 \%)$ & $44 / 73(58.3 \pm 2.3)^{\mathrm{b})}$ & $1.05 \pm 1.11^{\mathrm{b})}$ \\
\hline
\end{tabular}

Values in the parentheses are presented as mean \pm standard error of the mean.

${ }^{a, b}$ Different superscripts indicate values that differ significantly at $p<0.05$.

\section{Discussion}

This study demonstrated that the in vitro culture of mouse embryos under $5 \%$ to $2 \%$ oxygen concentration (the dynamic- $\mathrm{O}_{2}$ group) with a DCGS incubator system improved development of mouse pre- and peri-implantation embryos compared with low (5\%) and high (20\%) oxygen concentrations. We consistently found adverse effects of an atmospheric oxygen concentration (20\%) on mouse embryonic development during in vitro culture.

Oxygen concentration affects the rate of in vitro embryonic development and quality. The majority of modern IVF clinics have used $5 \%$ or $20 \%$ oxygen concentrations when culturing embryos under in vitro incubation. However, controversy still exists regarding whether a further reduction of oxygen concentration on day 3 after fertilization (the post-compaction stage) represents a physiological state that is more similar to in vivo oxygen conditions. This approach is based on the assumption that the physiological oxygen concentration is actually lower in the uterus (at approximately $2 \%$ ) than in the oviduct (at $5 \%-7 \%)[9-11,22,23]$.

In a previous report, bovine embryos were cultured at various oxygen concentrations $(0 \%, 1 \%, 2 \%, 4 \%$, and $7 \%)$, and these embryos displayed the highest blastocyst development rate under the $2 \%$ oxygen concentration condition [24]. Furthermore, Kaser et al. [27] ex- amined the blastocyst development potential of normal and abnormal embryos after culture under $2 \%$ or $5 \%$ oxygen concentrations from the post-compaction stage. Embryos cultured under a 2\% oxygen concentration displayed a higher blastulation rate and a higher utilization rate. However, in our results, the blastulation rate in the $5 \%$ to $2 \%$ oxygen concentration group was not significantly different from that in the $5 \%$ oxygen concentration group, although a signifcantly higher development rate was observed than in the $20 \%$ oxygen group. Similarly, Feil et al. [28] examined mouse embryo culture at a $7 \%$ oxygen concentration from the zygote stage embryo to the morula stage, and surviving morulae were then randomly cultured to the blastocyst stage at $2 \%, 7 \%$, or $20 \%$ oxygen. In the results of that study, no differences were found in the blastulation rate among these groups. In studies of human embryos, there were no significant differences in the development rate to the blastocyst stage or in the number of high-quality blastocysts when embryos were cryopreserved on day 3 and cultured to the blastocyst stage at $2 \%, 5 \%$, or $20 \%$ oxygen [29]. This study applied dynamic oxygen conditions, decreasing from $5 \%$ to $2 \%$; these concentrations were determined through careful review of many previous studies and a brief preliminary experiment (data not shown).

Oxygen concentrations during the in vitro culture of embryos, from $20 \%$ to lower levels, were related to embryonic development and embryo quality. The blastulation rate and the total cell number of blastocysts were related to subsequent fetal viability [30]. Many studies have shown that low oxygen concentrations were beneficial to embryonic development compared to higher oxygen concentrations $[13,17,19,24,31-36]$. Those previous studies used a single oxygen concentration, as the automatic oxygen-changing incubator system used in our study was not available. A recent study conducted by De Munck et al. [37] compared the effects of direct or gradual exposure to $2 \%$ or $5 \%$ oxygen on the in vitro culture of human post-compaction embryos. No significant difference was found in blastulation and the good-quality embryo rate between the $2 \%$ and $5 \%$ oxygen con- 
centrations.

Our study, unlike most previous studies, developed and applied a novel DCGS incubator to automatically change the oxygen concentration. This experiment incorporated sequential exposure to a $5 \%$ oxygen concentration from day 1 to day 3 (the pre-compaction stage), and then to a $2 \%$ oxygen concentration from day 3 to day 5 (the post-compaction stage), which was determined in consideration of the physiological oxygen conditions of the female reproductive tract. This DCGS incubator system may be advantageous in that it can automatically change the oxygen concentration from $5 \%$ to $2 \%$ without any interference or adaptation time. In this study, the total cell number of blastocysts was significantly higher in the dynamic- $\mathrm{O}_{2}$ (5\% to $2 \%$ ) group compared with the low- $\mathrm{O}_{2}(5 \%)$ and high- $\mathrm{O}_{2}(20 \%)$ groups. The apoptotic index of the dynamic- $\mathrm{O}_{2}$ (5\% to $2 \%$ ) group was also the lowest of the three groups. In contrast, Yang et al. [29] reported that there was no significant difference between the total cell number of cultured blastocysts in 2\% and 5\% oxygen after the post-compaction stage compared to the $20 \%$ oxygen condition. The difference between that study and our study is the oxygen concentration of the pre-compaction stage from day 1 to day 3 (20\% vs. $5 \%$, respectively). It can be assumed that 20\% oxygen induced irreversible damage to the blastocysts via abundant ROS products.

Blastocyst outgrowth has been used as an in vitro model for the implantation of developing embryos in utero. It could be applied for various studies of trophoblasts physiology and implantation competence in peri-implantation embryos $[26,38,39]$. Aspects of the in vitro trophoblastic outgrowth model, such as activation and spread, are related to proliferation and invasion in in vivo implantation processes [40-43]. In addition, morphokinetics and metabolism, as evidenced by the trophoblastic outgrowth assay, are correlated with viability and implantation potential $[44,45]$. In this study, the outgrowth rate after 3 days of culture of blastocysts in the dynamic- $\mathrm{O}_{2}(5 \%$ to $2 \%)$ and low- $\mathrm{O}_{2}(5 \%)$ groups was superior to that in the high- $\mathrm{O}_{2}(20 \%)$ group. Additionally, the mean trophoblastic spread area of the dynamic- and low- $\mathrm{O}_{2}$ groups was significantly wider than that of the high- $\mathrm{O}_{2}$ group. These results show that preimplantation embryos cultured under dynamic $\mathrm{O}_{2}$ (5\% to $2 \%$ ) concentrations could have a greater viability and implantation potential. There is a limitation of this study to not fully excluding exposure to $20 \%$ oxygen in the air during sampling and handling of mouse embryos. There is a limitation of this study to not fully excluding exposure to $20 \%$ oxygen in the air during sampling and handling of mouse embryos. Nonetheless, in the in vitro culture of preimplantation embryos, optimized oxygen concentrations similar to those in vivo should be considered as a necessary factor for improving culture conditions.

In conclusion, the results of this study demonstrate the beneficial effects of dynamic oxygen concentrations, shifting from $5 \%$ to $2 \%$ on day 3. This may be related to the physiological conditions experienced by preimplantation embryos in the uterus. We recommend that future studies include more samples, and we posit that embryo transfer in vivo is also required to fully investigate the implantation potential of in vitro cultured embryos. Additionally, more research should explore the application of other oxygen conditions during embryo culture. We suggest that the optimized and dynamic changing of oxygen concentrations with the novel DCGS incubator could improve the developmental competence of in vitro cultured embryos in human IVF/embryo transfer programs.

\section{Conflict of interest}

No potential conflict of interest relevant to this article was reported.

\section{ORICD}

Seung-Chan Lee https://orcid.org/0000-0003-2100-3000 Jaewang Lee https://orcid.org/0000-0001-6801-7149 Ho-Chul Seo https://orcid.org/0000-0002-8156-2317 Jin Hyun Jun ～https://orcid.org/0000-0001-9898-4471 Kyoo Wan Choi https://orcid.org/0000-0003-1998-2684

\section{Author contributions}

Conceptualization: JHJ, KWC. Data curation: SCL, JL, HCS. Method ology: SCL, JL, HCS, JHJ. Project administration: JHJ, KWC. Writing - original draft: SCL, JHJ. Writing - review \& editing: JL, HCS, KWC.

\section{References}

1. Leese HJ. Metabolic control during preimplantation mammalian development. Hum Reprod Update 1995;1:63-72.

2. Thompson JG, Partridge RJ, Houghton FD, Cox Cl, Leese HJ. Oxygen uptake and carbohydrate metabolism by in vitro derived bovine embryos. J Reprod Fertil 1996;106:299-306.

3. Goto Y, Noda Y, Mori T, Nakano M. Increased generation of reactive oxygen species in embryos cultured in vitro. Free Radic Biol Med 1993:15:69-75.

4. Harvey AJ, Kind KL, Thompson JG. REDOX regulation of early embryo development. Reproduction 2002;123:479-86.

5. Takahashi M. Oxidative stress and redox regulation on in vitro development of mammalian embryos. J Reprod Dev 2012;58:19.

6. Johnson MH, Nasr-Esfahani MH. Radical solutions and cultural problems: could free oxygen radicals be responsible for the impaired development of preimplantation mammalian embryos 
in vitro? Bioessays 1994;16:31-8.

7. Yang HW, Hwang KJ, Kwon HC, Kim HS, Choi KW, Oh KS. Detection of reactive oxygen species (ROS) and apoptosis in human fragmented embryos. Hum Reprod 1998;13:998-1002.

8. Mastroianni L Jr, Jones R. Oxygen tension within the rabbit fallopian tube. J Reprod Fertil 1965;9:99-102.

9. Maas DH, Storey BT, Mastroianni L Jr. Oxygen tension in the oviduct of the rhesus monkey (Macaca mulatta). Fertil Steril 1976; 27:1312-7.

10. Pierce GB, Parchment RE, Lewellyn AL. Hydrogen peroxide as a mediator of programmed cell death in the blastocyst. Differentiation 1991;46:181-6.

11. Fischer B, Bavister BD. Oxygen tension in the oviduct and uterus of rhesus monkeys, hamsters and rabbits. J Reprod Fertil 1993; 99:673-9.

12. Steptoe PC, Edwards RG, Purdy JM. Human blastocysts grown in culture. Nature 1971;229:132-3.

13. Umaoka Y, Noda Y, Narimoto K, Mori T. Effects of oxygen toxicity on early development of mouse embryos. Mol Reprod Dev 1992; 31:28-33.

14. Olson SE, Seidel GE Jr. Reduced oxygen tension and EDTA improve bovine zygote development in a chemically defined medium. J Anim Sci 2000;78:152-7.

15. Lequarre AS, Marchandise J, Moreau B, Massip A, Donnay I. Cell cycle duration at the time of maternal zygotic transition for in vitro produced bovine embryos: effect of oxygen tension and transcription inhibition. Biol Reprod 2003;69:1707-13.

16. Yuan YQ, Van Soom A, Coopman FO, Mintiens K, Boerjan ML, Van Zeveren $A$, et al. Influence of oxygen tension on apoptosis and hatching in bovine embryos cultured in vitro. Theriogenology 2003;59:1585-96.

17. Quinn P, Harlow GM. The effect of oxygen on the development of preimplantation mouse embryos in vitro. J Exp Zool 1978; 206:73-80.

18. Harlow GM, Quinn P. Foetal and placental growth in the mouse after pre-implantation development in vitro under oxygen concentrations of 5 and 20\%. Aust J Biol Sci 1979;32:363-9.

19. Gardner DK, Lane M. Alleviation of the'2-cell block' and development to the blastocyst of CF1 mouse embryos: role of amino acids, EDTA and physical parameters. Hum Reprod 1996;11:270312.

20. Karagenc L, Sertkaya Z, Ciray N, Ulug U, Bahceci M. Impact of oxygen concentration on embryonic development of mouse zygotes. Reprod Biomed Online 2004;9:409-17.

21. Wale PL, Gardner DK. Time-lapse analysis of mouse embryo development in oxygen gradients. Reprod Biomed Online 2010; 21:402-10.
22. Yedwab GA, Paz G, Homonnai TZ, David MP, Kraicer PF. The temperature, $\mathrm{pH}$, and partial pressure of oxygen in the cervix and uterus of women and uterus of rats during the cycle. Fertil Steril 1976;27:304-9.

23. Ottosen LD, Hindkaer J, Husth M, Petersen DE, Kirk J, Ingerslev HJ. Observations on intrauterine oxygen tension measured by fibre-optic microsensors. Reprod Biomed Online 2006;13:380-5.

24. Thompson JG, McNaughton C, Gasparrini B, McGowan LT, Tervit HR. Effect of inhibitors and uncouplers of oxidative phosphorylation during compaction and blastulation of bovine embryos cultured in vitro. J Reprod Fertil 2000;118:47-55.

25. Park JC, Kim JA, Kim DJ, Bae JG, Kim Jl, Rhee JH. Effect of human hydrosalpingeal fluid on the development of mouse embryo. Korean J Reprod Med 2010;37:125-34.

26. Kim J, Lee J, Kim SH, Jun JH. Coculture of preimplantation embryos with outgrowth embryos improves embryonic developmental competence in mice. Reprod Sci 2016;23:913-23.

27. Kaser DJ, Bogale B, Sarda V, Farland LV, Williams PL, Racowsky C. Randomized controlled trial of low (5\%) versus ultralow (2\%) oxygen for extended culture using bipronucleate and tripronucleate human preimplantation embryos. Fertil Steril 2018;109: 1030-7.

28. Feil D, Lane M, Roberts CT, Kelley RL, Edwards LJ, Thompson JG, et al. Effect of culturing mouse embryos under different oxygen concentrations on subsequent fetal and placental development. J Physiol 2006;572(Pt 1):87-96.

29. Yang Y, Xu Y, Ding C, Khoudja RY, Lin M, Awonuga AO, et al. Comparison of 2, 5, and $20 \% 02$ on the development of post-thaw human embryos. J Assist Reprod Genet 2016;33:919-27.

30. Lane M, Gardner DK. Differential regulation of mouse embryo development and viability by amino acids. J Reprod Fertil 1997; 109:153-64.

31. Pabon JE Jr, Findley WE, Gibbons WE. The toxic effect of short exposures to the atmospheric oxygen concentration on early mouse embryonic development. Fertil Steril 1989;51:896-900.

32. Thompson JG, Simpson AC, Pugh PA, Donnelly PE, Tervit HR. Effect of oxygen concentration on in-vitro development of preimplantation sheep and cattle embryos. J Reprod Fertil 1990;89: 573-8.

33. Li J, Foote RH. Culture of rabbit zygotes into blastocysts in protein-free medium with one to twenty per cent oxygen. J Reprod Fertil 1993;98:163-7.

34. Dumoulin JC, Meijers CJ, Bras M, Coonen E, Geraedts JP, Evers JL. Effect of oxygen concentration on human in-vitro fertilization and embryo culture. Hum Reprod 1999;14:465-9.

35. Hooper K, Lane M, Gardner DK. Reduced oxygen concentration increases mouse embryo development and oxidative metabo- 
lism. Theriogenology 2001;55:334.

36. Orsi NM, Leese HJ. Protection against reactive oxygen species during mouse preimplantation embryo development: role of EDTA, oxygen tension, catalase, superoxide dismutase and pyruvate. Mol Reprod Dev 2001;59:44-53.

37. De Munck N, Janssens R, Segers I, Tournaye H, Van de Velde $H$, Verheyen $\mathrm{G}$. Influence of ultra-low oxygen (2\%) tension on in-vitro human embryo development. Hum Reprod 2019;34:228-34.

38. Wang J, Mayernik L, Armant DR. Integrin signaling regulates blastocyst adhesion to fibronectin at implantation: intracellular calcium transients and vesicle trafficking in primary trophoblast cells. Dev Biol 2002;245:270-9.

39. Mardon H, Grewal S, Mills K. Experimental models for investigating implantation of the human embryo. Semin Reprod Med 2007;25:410-7.

40. Qin J, Takahashi Y, Isuzugawa K, Imai M, Yamamoto S, Hirai Y, et al. Regulation of embryo outgrowth by a morphogenic factor, epimorphin, in the mouse. Mol Reprod Dev 2005;70:455-63.

41. Hannan NJ, Paiva P, Meehan KL, Rombauts LJ, Gardner DK, Sal- amonsen LA. Analysis of fertility-related soluble mediators in human uterine fluid identifies VEGF as a key regulator of embryo implantation. Endocrinology 2011;152:4948-56.

42. Gonzalez IM, Martin PM, Burdsal C, Sloan JL, Mager S, Harris T, et al. Leucine and arginine regulate trophoblast motility through mTOR-dependent and independent pathways in the preimplantation mouse embryo. Dev Biol 2012;361:286-300.

43. Hsuuw YD, Chan WH, Yu JS. Ochratoxin a inhibits mouse embryonic development by activating a mitochondrion-dependent apoptotic signaling pathway. Int J Mol Sci 2013;14:935-53.

44. Lee YS, Thouas GA, Gardner DK. Developmental kinetics of cleavage stage mouse embryos are related to their subsequent carbohydrate and amino acid utilization at the blastocyst stage. Hum Reprod 2015;30:543-52.

45. Kim J, Kim SH, Jun JH. Prediction of blastocyst development and implantation potential in utero based on the third cleavage and compaction times in mouse pre-implantation embryos. J Reprod Dev 2017;63:117-25. 